\title{
Caractérisation et restitution vidéographique des ambiances urbaines
}

\author{
Gabriel Giraldo ${ }^{1, *}$, Myriam Servières ${ }^{1 *}$, Vincent Tourre $^{1 *}$, Valerio Signorelli ${ }^{2}$, et Aurore \\ Bonnet $^{3}$
}

${ }^{1}$ (AAU) Ambiances, Architectures, Urbanités, Équipe CRENAU ENSA Nantes 6 Quai François Mitterrand Nantes CEDEX 2, France - Équipe CRESSON ENSA Grenoble 60 Avenue de Constantine Grenoble CEDEX 2, France

${ }^{2}$ (CASA) Centre for Advanced Spatial Analysis, University College London, Gower Street, London, WC1E 6BT, Royaume-Uni

${ }^{3}$ Chercheuse indépendante

\begin{abstract}
Résumé. À la suite d'une immersion dans un espace public pour avoir notre propre perception du lieu et analyser les comportements des usagers, nous avons procédé à une simulation de phénomènes physiques pour mieux comprendre la nature des phénomènes remarqués par les usagers. Le ressenti des usagers a été ensuite restitué dans une vidéo descriptive dans le but de transmettre les éléments d'ambiance qui caractérisent l'espace étudié. Les réactions des spectateurs de la vidéo, usagers ou non-usagers de l'espace, montrent la pertinence des effets visuels choisis pour restituer les phénomènes physiques.
\end{abstract}

Mots-clés. Ambiances urbaines, Écologie humaine, Espace public, Simulation numérique, Rendu vidéo.

\begin{abstract}
After being immersed in a public space, in order to have our own perception of the place itself and to analyze users' behavior, we ran simulations of physical phenomena to better understand the nature and magnitude of the ones noticed by the users. Finally, a descriptive video has been used as a way to reveal the variety of users' experiences and to convey the peculiarities of the atmospheres of the chosen area. The feedback from spectators, users or non-users of the space shows the relevance of chosen visual effects to convey the physical phenomena.
\end{abstract}

Keywords. Urban Atmospheres, Human Ecology, Public Space, Digital Simulation, Video Rendering.

\footnotetext{
* Corresponding author: gabriel.giraldo@crenau.archi.fr
} 


\section{Introduction}

L'étude d'ambiances urbaines vise à grouper des approches d'ordre quantitatif (régies par les lois de la physique) et qualitatif (venant du vécu des espaces) (Péneau, 1990) pour la compréhension et l'analyse des facteurs climatiques, environnementaux et comportementaux qui sont générés dans et par l'espace. Cela intègre des considérations liées au confort spatial des usagers dans la ville et aux phénomènes physiques qui nourrissent la perception de l'individu qui est protagoniste et spectateur dans le déroulement quotidien de la vie urbaine (Lerner et Gehl, 2014). La réalité complexe des ambiances urbaines a son origine dans l'ensemble des facteurs physiques et environnementaux, le sujet singulier qui sent, ressent ou perçoit et l'interaction des sujets inclus dans l'espace (Augoyard, 2011; Thomas et al., 2014).

Nous nous sommes intéressés à l'étude des ambiances urbaines d'un lieu en partant d'une analyse parallèle de données recueillies avec des méthodes tant qualitatives que quantitatives, pour essayer d'identifier et de représenter les ambiances urbaines ressenties et les modes d'habiter des usagers de l'espace public étudié.

Au niveau de la restitution des expériences, nous nous appuyons sur la technique de la rotoscopie (Achemchame, 2013). Il s'agit donc de fusionner, selon l'optique du transect urbain (Tixier, 2011) les éléments observables et audibles capturés par la caméra avec ceux qui ne le sont pas.

\section{Description générale de la méthode}

La figure 1 décrit d'un point de vue global le processus suivi sur site et hors-site et ses différentes étapes. La première partie «caractérisation » (section 2.1) permet de déterminer et de qualifier les marqueurs d'ambiance de la zone étudiée, la deuxième partie « restitution » (section 2.2) permet de fusionner les deux types de caractérisations par le biais d'une vidéo et d'analyser les réactions des spectateurs.

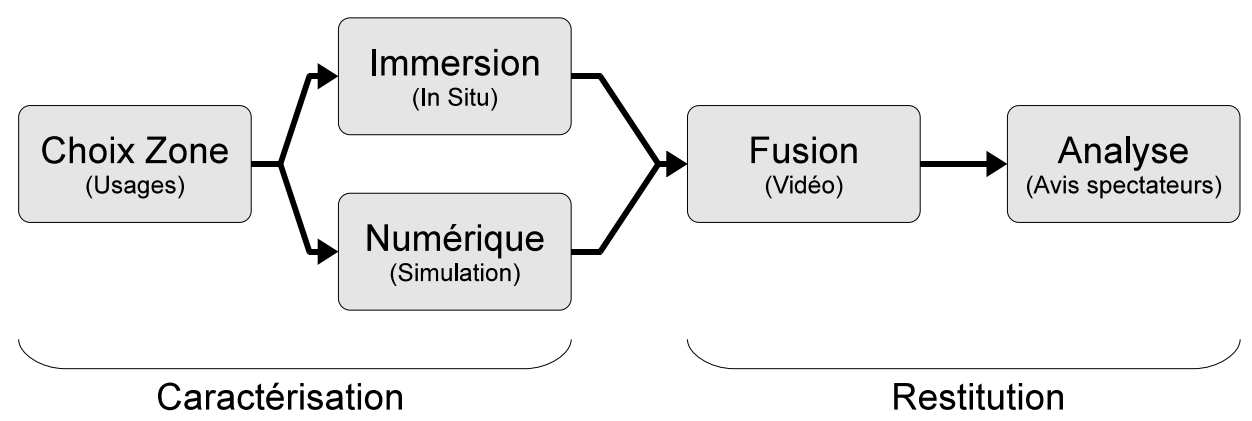

Figure 1. Processus de l'application de la méthode sur site et hors site. 


\subsection{Méthode de caractérisation des marqueurs d'ambiances, à la fois qualitatifs et quantitatifs}

Cette première étape consiste à répertorier les effets des phénomènes physiques sur la perception sensible produits dans l'espace. Il s'agit de faire ressortir l'essence même des sensations vécues par les usagers, pour comprendre et caractériser l'espace d'étude. Cette étape s'est basée sur les méthodes des parcours commentés (Thibaud, 2001), l'éthologie des espaces publics (Cosnier, 2001), l'observation récurrente (Amphoux, 2001) et les méthodes de mapping, tracing et tracking (Gehl et Svarre, 2013). Elle s'est déroulée en cinq temps.

D'abord, nous avons cherché la zone d'étude sur la base de son potentiel à accueillir divers usagers et de permettre le développement de leurs activités pendant différentes temporalités. Nous nous sommes intéressés à une zone qui peut offrir une diversité spatiale, sociale et fonctionnelle, et en même temps qui est soumise à l'incidence directe des phénomènes physiques qui peuvent influencer et caractériser les comportements des utilisateurs dans des zones spécifiques du même espace. Nous avions l'intention de trouver un lien entre ces phénomènes, les formes urbaines, et leurs répercussions dans les comportements et perceptions résultantes des usagers.

En second lieu, nous avons effectué une captation sensible/multisensorielle. C'est une approche d'immersion in situ pour découvrir l'espace et faire réagir nos sens aux différents évènements. Ainsi nous avons pu distinguer à chaque nouvelle visite les dynamiques réitératives qui ont pu donner des signes des origines d'un tel comportement (Thomas et al., 2014). En parallèle, notre attention s'est focalisée sur l'observation des usagers avec un regard d'ordre étho-antropologique (Cosnier, 2001) qui consiste à observer tous les comportements "naturels " des utilisateurs dans leur environnement quotidien en perturbant le moins possible ces comportements.

En troisième lieu, nous avons construit des expériences collectives partagées avec des usagers. La méthode du parcours commenté (Thibaud, 2001) a été mise en œuvre pour accompagner les usagers de l'espace dans une déambulation afin de découvrir leurs expériences au fur et à mesure qu'ils parcouraient l'espace.

La quatrième partie a consisté en une caractérisation de l'objet à capturer. Cela a été une étape d'analyse de chaque expérience individuelle pour découvrir les situations répétitives évoquées par les usagers, notamment les modes d'appropriation de l'espace en fonction des phénomènes physiques et sensibles existants.

Finalement, nous avons terminé par une étape de capture instrumentée des ambiances. Le moment de l'enregistrement avec un regard « orienté » où « le rapport entre observation et description s'inverse : il ne s'agit plus de décrire ce que l'on perçoit mais de rapporter les descriptions à ce qui est observable sur place » (Thibaud, 2001, p. 88). Nous avons classifié l'information en catégories selon le phénomène physique sur lequel elle attirait l'attention. L'ensemble de ces données a été analysé conjointement avec les résultats des simulations numériques des phénomènes physiques observés (aéraulique, ensoleillement). Ces simulations ont révélé les liens entre les phénomènes microclimatiques et la morphologie urbaine (Athamena, 2012) et ont ainsi quantifié objectivement les expériences des usagers in situ.

\subsection{Restitution des ambiances et analyse des effets transmis}

Notre parti pris est de produire une restitution des données acquises sur site et simulées numériquement via un rendu audio-visuel. Nous avons réassemblé l'information capturée en un seul élément structuré de telle façon qu'il puisse transmettre et mettre en évidence certains marqueurs à l'aide des techniques d'édition des vidéos telles que le compositing (Meigneux, 2012) et la rotoscopie (Achemchame, 2013). Elles nous ont permis de grouper 
les comportements réitératifs développés sur différentes temporalités et les communiquer sur une même scène ; et d'associer des objets numériques résultants des simulations sur des images réelles enregistrées du site, cela afin de restituer un ensemble cohérent de marqueurs d'ambiance.

Pour évaluer la restitution nous avons fait visionner la vidéo à deux groupes de personnes. L'un des groupes ne connaissait pas le lieu et a donné son avis sur les ambiances traduites par la vidéo ; le deuxième groupe était usager de l'espace et devait faire un retour sur le fait qu'il identifiait ou non son expérience vécue.

\section{Caractérisation de l'espace}

\subsection{Choix de la zone d'étude}

Nous avons choisi l'île Gloriette de Nantes car elle est soumise à l'incidence directe de phénomènes physiques prégnants qui peuvent caractériser les comportements des utilisateurs, par exemple: les courants du vent provenant de la Loire, les sons de l'hélicoptère et les ambulances du CHU, et les masques solaires des bâtiments sur l'espace public. Cet espace se localise à l'ouest du CHU de Nantes (figure 2). L'espace public est divisé en deux zones, le passage de la rue Bias et une «place » située devant la bibliothèque universitaire (BU). Après avoir été une voie de desserte, la rue Bias a été aménagée en passage piétonnier et sert de connexion entre le CHU et la BU. Dans son prolongement cet espace aménagé débouche sur un espace dégagé qui a un aspect brut, une "place » sans nom et sans signes d'aménagement récents (graviers, blocs de béton).
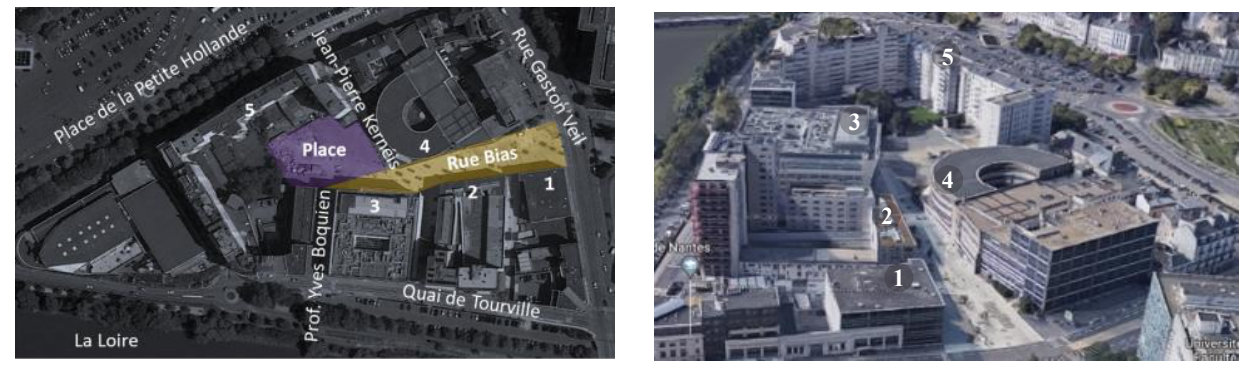

Figure 2. Zone d'étude (sources Google Earth et Google Maps). À l'intérieur de l'îlot quelques constructions récentes (l'amphithéâtre Kerneis (1), un logement étudiant (2) et la BU (3)), coexistent avec des constructions plus anciennes et détériorées (un parking (4) et un logement collectif (5)).

\subsection{Approche immersive}

Cette étape s'est déroulée lors de visites sur la zone d'étude le 26 avril et le 27 mai 2016. Lors des premières visites, nous avons vécu l'espace en immersion, en usager. Dans le même temps, nous avons observé et relevé les comportements habituels des usagers tout au long de la journée. Les visites suivantes ont été destinées à échanger avec les usagers et à l'enregistrement des situations réitératives.

\subsubsection{Observations}

Les journées ont été contrastées au niveau météorologique et dans le comportement des usagers. La première journée (26 avril) a été caractérisée par un ciel couvert et un vent fort. La « place » est restée presque déserte pendant la matinée. La rue Bias est utilisée comme 
une zone de transit pour arriver à la $\mathrm{BU}$, néanmoins personne ne reste sur ses bancs. Les courants provenant de la Loire ont une forte incidence dans le comportement aéraulique du passage. Un premier endroit de rassemblement est identifié : la porte de la $\mathrm{BU}$, où les étudiants sortent pour la pause-café. Après avoir suivi quelques usagers qui marchaient vers la rue Professeur Yves Boquien, un deuxième lieu est identifié : l'entrée du parking de la BU ; cet espace, interdit aux piétons, est néanmoins utilisé car protégé du vent. Dans la rue Bias, la plupart des gens marchent du côté ensoleillé du passage malgré la cohabitation avec les voitures (figure 3, à gauche), alors que le côté exclusivement piétonnier non-ensoleillé reste moins fréquenté. Dans la deuxième journée (27 mai), où la présence du soleil a été beaucoup plus forte, les usagers s'éloignent de l'entrée de la BU pour leurs pauses et la « place » devient l'espace préféré pour s'installer (figure 3, à droite).
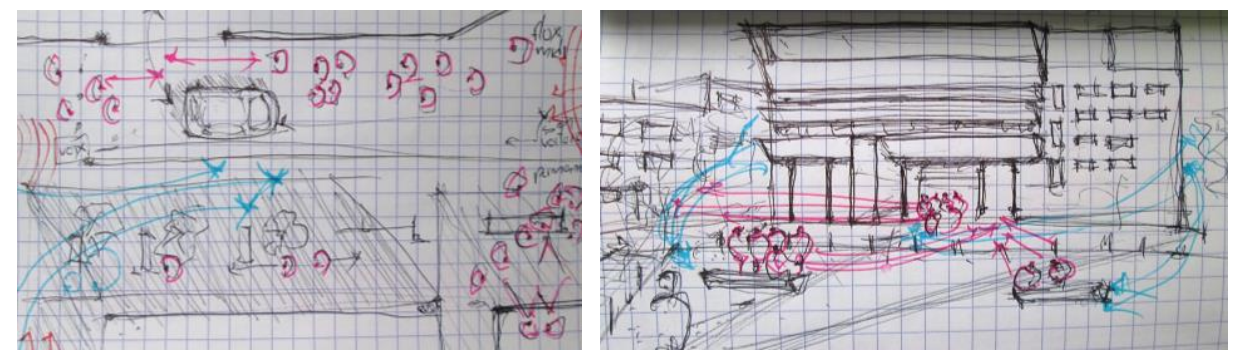

Figure 3. Incidence du vent et du soleil, observés sur les comportements des usagers.

\subsubsection{Paroles d'usagers}

Les interviews préliminaires des usagers installés dans l'espace public relatives à leur perception de l'espace affinent les observations faites précédemment. De plus, ils étaient conscients de leur appropriation de l'espace en fonction des conditions climatiques: "Quand il fait beau on se pose sur les bancs [de la place] pour...pour manger....- Pour jouer....- Profiter du soleil un peu », " ici [la porte de la BU] à l'abri du vent, quand il fait froid, qu'il n'y a pas de rayons de soleil on se met là, et quand il y a un peu de rayons de soleil on se met là ou là-bas [la place] " "Le vent? Là-bas il y a le petit garage [l'entrée du parking] (...) ça coupe du vent - ça pue bien, mais au moins c'est à l'abri du vent ».

Ensuite, nous avons appliqué la méthode des parcours commentés (Thibaud, 2001) avec les usagers tout au long de la rue Bias et le passage Jean-Pierre Kernéis. Les principales remarques ont été liées au contraste de l'environnement bâti, le fait de partager le chemin avec les voitures, les sons des oiseaux, la faible taille des arbres, les tonalités neutres, le flux du vent et le manque d'abri : « il y a des voitures qui passent souvent, c'est dangereux pour les passants (...), parce qu'il y a des confrontations parfois », "lorsqu'il fait beau, on entend souvent le bruit des oiseaux qui arrivent très souvent à côté du fleuve ", « les pins sont vraiment tous petits petits, donc, c'est un peu moche, quand ils seront un peu plus grands ça sera plus joli », "il y a un jeu de contraposition entre des bâtiments, (...) tu vois un bâtiment qui est délabré d'un côté et de l'autre un qui est un peu plus neuf, et celui qui est plus neuf s'oppose aussi à la BU qui a un côté beaucoup plus statutaire, il s'impose notamment pour le nombre d'étages", "C'est vrai que le passage là, même quand il y a du soleil, il est plus sombre...- Et [il y a] du vent...-Alors que là [la place], quand il y a du soleil ça passe bien, voilà », sont quelques exemples des remarques des usagers. En conclusion de cette étape, nous pouvons distinguer des espaces destinés au rassemblement et d'autres destinés au passage, en fonction des conditions climatiques. Avec un ciel couvert, la porte de la BU et l'entrée au parking deviennent plus utilisés, tandis que dans une situation ensoleillée, la « place » offre les conditions plus favorables. 


\subsection{Approche numérique}

Suite aux expériences vécues dans le site, nous avons construit une maquette numérique 3D afin d'analyser les effets principalement décrits dans le lieu : le vent et le soleil (figure 4). Les phénomènes sonores ont été peu abordés lors des discussions certainement en raison de l'accoutumance des usagers, et donc n'ont pas été retenus pour effectuer des simulations. Nous avons simulé le comportement du vent sur l'extension de Code_Saturne pour SOLENE ${ }^{1}$ selon sa direction prédominante nord-ouest et de l'ouest. Nous constatons des effets de décélération du vent à la porte d'entrée de la BU dus à l'addition du volume cylindrique (figure 4 , gauche, (1)), qui protège les usagers du vent. De même, il y a une réduction de la vitesse du vent dans l'entrée du parking (figure 4, gauche, (2)), due à la confrontation des courants d'air provenant de la rue Professeur Yves Boquien et de la rue Bias. Nous avons effectué des simulations de l'ensoleillement de l'espace sur SOLENE pour recréer le comportement du 27 mai (figure 4, droite). Nous trouvons que le pourcentage moyen d'ensoleillement pendant les heures d'exposition est de 75,21\% pour la «place » tandis qu'il n'est que de 56,82\% pour la rue Bias. Ce faible ensoleillement ajouté à la haute incidence du vent permet une corrélation des résultats de simulation avec les expériences des usagers in situ.
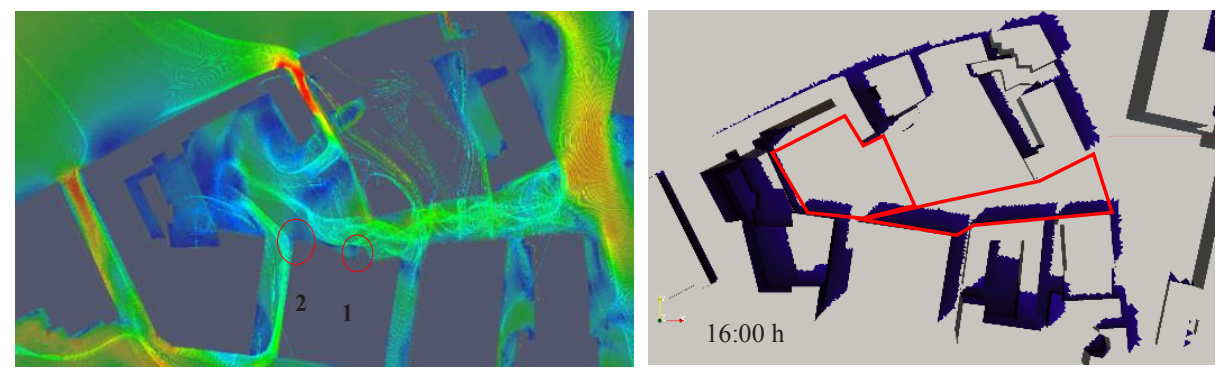

Figure 4. Simulation aéraulique avec Code_Saturne (à gauche), solaire avec SOLENE (à droite).

\section{Restitution et analyse}

\subsection{Restitution des marqueurs d'ambiances par la vidéo}

La vidéo ${ }^{2}$, comme moyen de restitution d'expériences in situ, nous permet de mettre en évidence quelques phénomènes sensibles et physiques qui influencent les modes d'occupation de l'espace étudié. Elle nous permet aussi de faire une association entre les données résultantes des observations, des interviews et des simulations numériques. Nous avons organisé ce rendu en cinq temps qui groupent les éléments sonores et visuels qui caractérisent l'espace :

- L'espace où l'on doit être : nous proposons une vue parallèle du même espace sous des conditions climatiques opposées (figure 5). Nous manipulons la coloration des photographies afin de mettre en relief l'effet climatique cherché. Après, une première matérialisation du vent, en indiquant sa présence et direction, nous captons l'attention

\footnotetext{
${ }^{1}$ «Ensemble d'outils logiciels de simulation du microclimat urbain (...) » SOLENE |Équipe CRENEAU. Consulté le 4 janvier 2018, à l'adresse http://aau.archi.fr/crenau/solene/
}

${ }^{2}$ Un espace habité, vidéo consultée sur https://www.youtube.com/watch?v=chVIHBOyZkE 
du spectateur sur un espace de faible fréquentation piétonne à cause de sa basse exposition solaire.

- Un espace plus abrité : nous présentons un passage de transition, où il y a une haute incidence aéraulique. Nous exposons l'effet du vent en ajoutant les résultats des simulations aérauliques aux images réelles du site (figure 6, gauche), en cherchant ainsi à exposer le comportement du vent ressenti dans l'espace.

- On suit le soleil : avec l'apparition du soleil les usagers se déplacent vers la tache lumineuse. L'entrée de la BU, présentée simultanément pendant différents moments de la journée, montre ses flux de déplacement naturels par compositing.

- C'est moche ...mais... : nous montrons à la façon d'un dessin animé la perception d'un espace qui, malgré son apparence visuelle, devient le plus utilisé. Nous profitons de cette représentation pour mettre en évidence les endroits détériorés par le passage du temps avec la coloration sépia.

- Traversées : nous mettons l'accent sur le fait que les usagers n'utilisent pas l'un des passages aménagés et ils traversent la «place» en fonction de leurs besoins.
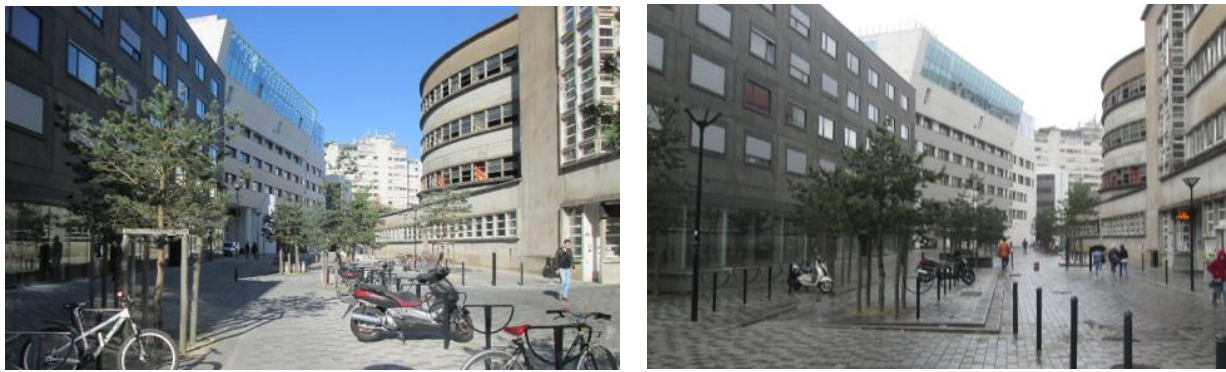

Figure 5. Même espace sous deux différentes conditions climatiques.
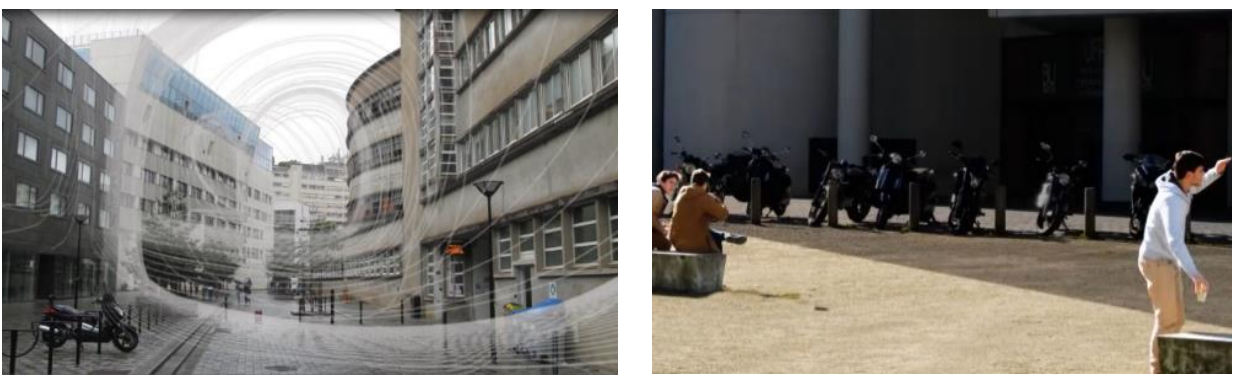

Figure 6. Résultats des simulations numériques surimposées aux images réelles ; aérauliques à gauche, solaires à droite.

\subsection{Analyse des réactions des spectateurs}

\subsubsection{Spectateurs qui n'habitent pas l'espace}

Nous avons fait évaluer la restitution vidéo des ambiances par sept personnes. Après leur avoir montré la vidéo, nous leur avons demandé leurs perceptions de l'espace en ayant des images de la vidéo imprimées comme support visuel lors de la discussion.

Globalement, les éléments les plus évoqués sont la façon dont le concepteur a conçu l'espace et la manière dont les usagers l'habitent, qui semblent contradictoires. À savoir, au 
lieu d'offrir des zones attractives ou des zones pour profiter du beau temps à l'extérieur, l'espace devient un endroit où les gens « survivent ». Ensuite, ils ont évoqué l'intention de montrer la pluralité de cet espace soumis à différents temporalités et conditions climatologiques. Ils ont repéré les vêtements des usagers et les ont associés à une saison.

Chaque séquence a ses aspects saillants. Dans l'espace où on doit être, les spectateurs ont principalement évoqué l'intention de présenter l'espace ensoleillé ou ombragé, qui a été renforcé lorsque l'ombre est matérialisée par le suivi du parcours d'un usager. Ensuite, ils notent la caractéristique de l'espace à être très exposé au niveau aéraulique et les bâtiments qui masquent le soleil. Dans la séquence Un espace plus abrité, toutes les personnes interviewées ont bien reçu le message de la forte influence du vent dans l'espace et ont aimé le fait de croiser les résultats de simulations avec les images de l'espace et les discours des usagers. Elles ont aussi remarqué et manifesté un sentiment d'étonnement quand elles se sont rendues compte que les étudiants se mettent dans un parking pour se protéger du vent. Dans On suit le soleil, les spectateurs ont bien remarqué les moments où les gens se placent juste dans la limite ombre/lumière, pour profiter du soleil. Les mouvements cumulatifs de l'entrée de la BU ont bien été mentionnés. C'est moche...mais... est la séquence où les opinions ont été les plus contrastées. Deux personnes parmi les interviewés ont mentionné que la coloration sépia utilisée rendait plus agréable l'espace et que cela contrastait avec le récit de l'étudiant. Les autres ont estimé que l'effet correspondait bien à l'idée que l'espace était moche et qu'il ne donne pas envie d'y rester. Enfin, dans la séquence Traversées, ils ont évoqué le fait que les gens préfèrent éviter le chemin conçu par l'urbaniste et en prendre un autre pour gagner quelques secondes dans leur parcours vers la BU.

\subsubsection{Spectateurs qui habitent l'espace}

Pour connaître les réactions des usagers qui habitent l'espace nous leur avons diffusé la vidéo des expériences par des réseaux sociaux et avec des nouvelles visites sur site. Ils ont remarqué le fait que les endroits et les phénomènes exprimés dans la vidéo correspondaient à leur expérience quotidienne, en mettant l'accent principalement sur l'influence du vent, et l'appropriation de la " place ». Bien que, de façon très générale, les spectateurs aient évoqué avoir identifié leurs comportements et perceptions de l'espace, nous regrettons de n'avoir pu obtenir des réponses plus spécifiques et différenciées par séquences qui auraient pu être très enrichissantes pour l'étude.

\section{Discussion et conclusions}

\subsection{Discussion}

Nous avons pu vivre, et puis nous avons tenté de recréer les états changeants des ambiances urbaines de l'île Gloriette, à savoir un phénomène résultant de l'addition de différents facteurs sensibles et physiques qui mettent en évidence la complexité des ambiances urbaines. Cependant ces résultats peuvent aussi être affectés par des facteurs saisonniers, il serait donc nécessaire reproduire ces expériences pendant des périodes variées, d'autres saisons ou des situations nocturnes. Ce type d'approches croisées, qui s'intéressent à la compréhension des phénomènes d'ambiance, peuvent beaucoup apporter à la conception architecturale et urbaine pour améliorer l'expérience vécue des usagers.

De façon générale, le moyen de restitution choisi a permis de transmettre correctement l'ensemble des perceptions des usagers. Le fait de rendre visibles les éléments cachés comme le vent ou de mettre en évidence les attitudes des usagers permet de mieux 
comprendre les dynamiques qui se déroulent dans un environnement inconnu. Ainsi des personnes qui n'habitent pas l'espace, après avoir vu la vidéo, ont partagé les perceptions des personnes qui l'expérimentent dans leur quotidien. Certains choix de représentation se sont révélés particulièrement pertinents, en particulier la surimposition de résultats de simulation aéraulique sur une prise de vue réelle pour traduire une exposition au vent. Néanmoins, la représentation des divers phénomènes sur la vidéo est le résultat des références culturelles et personnelles des auteurs, ce qui n'assure pas la compréhension universelle des éléments transmis. Ces choix introduisent deux biais : la compréhension du phénomène exprimé et la compréhension des effets produits par le phénomène sur le spectateur. Par exemple l'effet sépia qui a été compris comme donnant un effet agréable à la perception du lieu pour certains spectateurs alors que l'effet recherché était contraire.

Enfin, cette recherche montre qu'il est nécessaire de revenir à l'échelle de l'utilisateur qui devra affronter et assumer le résultat des choix des architectes ou urbanistes, qui eux se déplacent constamment parmi les différentes échelles de la ville.

\subsection{Conclusions}

Globalement, nos intentions initiales ont été bien reçues par les spectateurs qui n'habitent pas l'espace, sauf pour l'effet de coloration sépia qui a généré des divergences dans la réception du message. Nous avons pu mettre en pratique et en évaluation divers éléments tels que les approches sensibles, les approches numériques, la captation et ensuite la restitution vidéographique. Nous sommes conscients qu'il ne s'agit que de l'un des moyens de restitution possibles. Cette démarche montre l'intérêt de rendre visibles les éléments cachés dans un environnement urbain pour traduire des marqueurs d'ambiance urbaine. Cette représentation pourrait être aussi abordée avec les méthodes d'affichage utilisant la réalité mixte qui permettent d'afficher des objets virtuels dans un environnement réel et offrent la possibilité d'une interaction observateur/environ-nement. De tels outils pourraient croiser un ressenti sur le site et un affichage d'indicateurs d'ambiances issus d'enquêtes ou de simulations.

\section{Bibliographie}

Achemchame, J. (2013). Quand le cinéma ne suffit pas, la rotoscopie au service d'une adaptation cinématographique. À Scanner Darkly par Richard Linklater, d'après Philip K. Dick. Miranda. Revue pluridisciplinaire du monde anglophone / Multidisciplinary peer-reviewed journal on the English-speaking world, (8).

https://doi.org/10.4000/miranda.3344

Amphoux, P. (2001). L'observation récurrente. In L'espace urbain en méthodes (pp. 153-169). Marseille : Parenthèses.

Athamena, K. (2012, octobre 11). Modélisation et simulation des microclimats urbains : Étude de l'impact de la morphologie urbaine sur le confort dans les espaces extérieurs Cas des éco-quartiers. Thèse ECN.

Augoyard, J.-F. (2011). Faire une ambiance. Bernin : À la croisée.

Cosnier, J. (2001). L'éthologie des espaces publics. In L'espace urbain en méthodes (pp. 13- 28). Marseille : Parenthèses.

Gehl, J., et Svarre, B. (2013). How to Study Public Life. Washington : Island press.

Lerner, J., et Gehl, J. (2014). Urban acupuncture : celebrating pinpricks of change that enrich city life. Washington: Island press.

Meigneux, G. (2012). Le compositing : l'expérience de la durée et l'émancipation de l'observateur. In Ambiances in action (pp. 105-110). Grenoble : Réseau international Ambiances : École d'architecture de Grenoble. 
Peneau, J. P. (1990). Modélisation numérique des ambiances urbaines. In IMARA 1990 : Image Animée et Représentation Architecturale (pp. 31 - 37). Bordeaux: École d'architecture de Bordeaux.

Thibaud, J.-P. (2001). La méthode des parcours commentés. In L'espace urbain en méthodes (pp. 79- 99). Marseille: Parenthèses.

Thomas, R., et al. (2014). MUSE - Les énigmes sensibles des mobilités urbaines contemporaines. (Report, Recherche ANR, Programme «Espace et Territoire » ANR-10ESVS-013-01). Grenoble, Centre de recherche sur l'espace sonore et l'environnement urbain.

Tixier, N. (2011). L'ambiance est dans l'air : la dimension atmosphérique des ambiances architecturales et urbaines dans les approches environnementales. Grenoble : CRESSON. 